\title{
Strain modification of epitaxial perovskite oxide thin films using structural transitions of ferroelectric $\mathrm{BaTiO}_{3}$ substrate
}

\author{
M. K. Lee, T. K. Nath, and C. B. Eom ${ }^{\text {a) }}$ \\ Department of Materials Science and Engineering, University of Wisconsin-Madison, \\ Madison, Wisconsin 53706 \\ M. C. Smoak and F. Tsui \\ Department of Physics and Astronomy, University of North Carolina, Chapel Hill, North Carolina 27599
}

(Received 20 April 2000; accepted for publication 5 October 2000)

\begin{abstract}
Effects of induced biaxial strain on the electrical transport and magnetic properties of epitaxial thin films of $\mathrm{SrRuO}_{3}$ and $\mathrm{La}_{0.67} \mathrm{Sr}_{0.33} \mathrm{MnO}_{3}$ by structural transitions of ferroelectric $\mathrm{BaTiO}_{3}$ substrates have been studied. Large jumps of electrical resistivity $\left(\sim 5 \%\right.$ in $\mathrm{SrRuO}_{3}$ and $\sim 12 \%$ in $\left.\mathrm{La}_{0.67} \mathrm{Sr}_{0.33} \mathrm{MnO}_{3}\right)$ and low field magnetization $\left(\sim 70 \%\right.$ in $\left.\mathrm{La}_{0.67} \mathrm{Sr}_{0.33} \mathrm{MnO}_{3}\right)$ have been observed in the films at the structural transition temperatures of $\mathrm{BaTiO}_{3}$ substrate. The hysteretic jumps are reproducible through many thermal cycles, and they can be attributed to strain effects induced by the substrate. The use of phase transitions of ferroelectric substrates to manipulate lattice strain of epitaxial thin film heterostructures can be a useful way to modify the properties of perovskite oxides. (C) 2000 American Institute of Physics. [S0003-6951(00)01848-9]
\end{abstract}

A wide range of experimental studies of heteroepitaxial perovskite thin films indicate that lattice strain plays an important role in determining the physical properties of these films. For instance, strain has been shown to enhance superconducting transition temperature of $\mathrm{La}_{2}{ }_{x} \mathrm{Sr}_{x} \mathrm{CuO}_{4}$ thin films. ${ }^{1-3}$ Strain has also been shown to control the magnetoresistance and magnetic anisotropy effects in colossal magnetoresistive (CMR) manganite thin films. ${ }^{4,5}$ It is also known to suppress the Curie temperature and saturation magnetization of $\mathrm{SrRuO}_{3}$ thin films. ${ }^{6}$ Therefore, altering properties of novel perovskite oxide materials by lattice strain is very interesting not only for understanding solid state phenomena but also for potential device applications. Strain states in epitaxial heterostructures have been varied by using substrates with different lattice mismatch, and by varying film thickness. It is difficult to study intrinsic strain effects systematically using these methods, since many other variables such as lattice relaxation, stoichiometry, ${ }^{7}$ crystalline quality, and microstructure ${ }^{8}$ are all difficult to discriminate from sample to sample.

In this letter, we describe an approach to modify epitaxial constraints in epitaxial perovskite thin films using structural transitions of ferroelectric $\mathrm{BaTiO}_{3}$ substrates. Large biaxial strain can be produced at the transition temperatures, hence modifying properties and isolating the effects of strain. Initial study of epitaxial thin films of ferromagnetic $\mathrm{SrRuO}_{3}$ and $\mathrm{CMR} \mathrm{La}_{0.67} \mathrm{Sr}_{0.33} \mathrm{MnO}_{3}$ (LSMO) has been carried out using this method. The $\mathrm{SrRuO}_{3}$ and LSMO thin films were grown on $\mathrm{BaTiO}_{3}$ substrates by $90^{\circ}$ off-axis sputtering at 600 and $750{ }^{\circ} \mathrm{C}$, respectively. Room temperature four-circle $\mathrm{X}$-ray diffraction experiments were performed to measure the in-plane and out-of-plane lattice parameters.

$\mathrm{BaTiO}_{3}$ crystals exhibit three distinct structural phase transitions, ${ }^{9}$ as they are illustrated in the temperature depen-

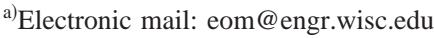

dent lattice parameters shown in Fig. 1. During cooling from high temperature, a $\mathrm{BaTiO}_{3}$ crystal passes through a cubic to tetragonal transition at $393 \mathrm{~K}$, then a transition to monoclinic structure at $278 \mathrm{~K}$, and finally to rhombohedral structure at 183 K. ${ }^{9}$ Room temperature $\mathrm{x}$-ray diffraction experiments show that the $\mathrm{BaTiO}_{3}$ substrates generally exhibit both (100) and (001) tetragonal domains, as one would expect. However, in this letter we report results only on films grown on pure (100) or (001) oriented $\mathrm{BaTiO}_{3}$ substrates for more quantitative discussions on strain effects. Out-of-plane and in-plane lattice parameters of the films have been measured by normal and grazing incidence x-ray diffractions at room temperature, and the corresponding strains, $\epsilon_{x x}, \epsilon_{y y}$, and $\epsilon_{z z}$, have been determined. The results are listed in Table I. As shown in Table I, both $1000 \AA \mathrm{SrRuO}_{3}$ and $500 \AA \mathrm{LSMO}$ epitaxial films exhibit partial strain relaxation at room temperature owing to their large lattice mismatch with $\mathrm{BaTiO}_{3}$ substrate (Fig. 1). However, it is reasonable to assume that

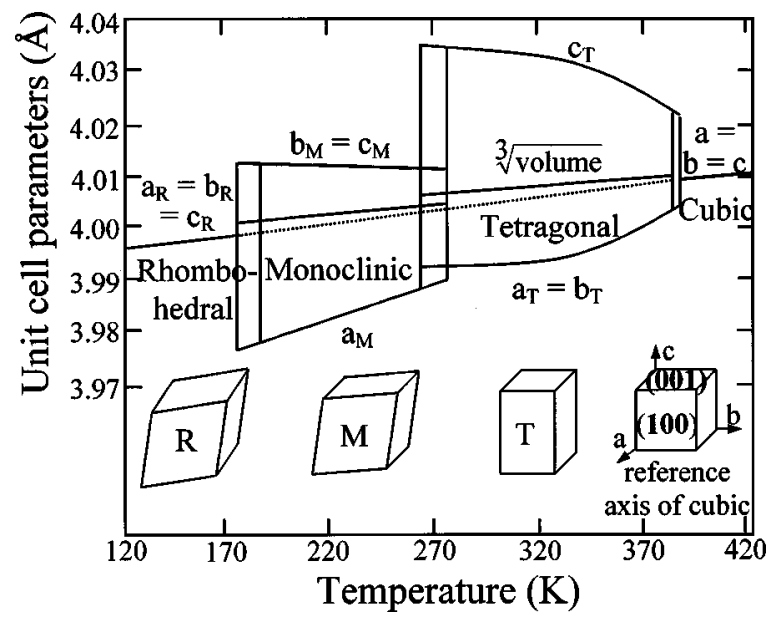

FIG. 1. Variation of lattice parameters as a function of temperature of $\mathrm{BaTiO}_{3}$ crystal. The letters, "T,', " $\mathrm{M}$," and " $\mathrm{R}$ ', represent tetragonal, monoclinic, and rhombohedral phases, respectively. 
TABLE I. Room temperature lattice parameters and corresponding strains of a $1000 \AA$ SRO film and a $500 \AA$ LSMO film.

\begin{tabular}{lcccccc}
\hline \hline & \begin{tabular}{c} 
Lattice \\
mismatch $^{\mathrm{a}}$ \\
\multicolumn{1}{c}{ Film }
\end{tabular} & $\begin{array}{c}\text { In-plane } \\
(\%)\end{array}$ & $\begin{array}{c}\text { Out-of- } \\
\text { plane } \\
(\AA)\end{array}$ & $\begin{array}{c}\boldsymbol{\epsilon}_{x x} \\
(\%)\end{array}$ & $\begin{array}{c}\boldsymbol{\epsilon}_{y y} \\
(\%)\end{array}$ & $\begin{array}{c}\boldsymbol{\epsilon}_{z z} \\
(\%)\end{array}$ \\
\hline $\mathrm{LSMO} /(001) \mathrm{BTO}$ & +3.24 & 3.94 & 3.84 & +1.50 & +1.50 & -1.03 \\
$\mathrm{SRO} /(100)$ & +1.75 & $3.92^{\mathrm{b}}$ & 3.92 & -0.25 & +0.51 & -0.25 \\
$\mathrm{BTO}$ & & 3.95 & & & & \\
\hline \hline
\end{tabular}

${ }^{\text {a }}$ Values are obtained from the bulk pseudocubic unit cells of $\mathrm{SrRuO}_{3}(a$ $=3.93 \AA)$ and $\mathrm{La}_{0.67} \mathrm{Sr}_{0.33} \mathrm{MnO}_{3}(a=3.88 \AA)$ and the cubic phase $(a=4.01$ $\AA$ ) of $\mathrm{BaTiO}_{3}$ substrate (Fig. 1).

${ }^{\mathrm{b}}$ Two in-plane lattice parameters correspond to a $0.3 \%$ contraction along [010] direction and a $0.7 \%$ elongation along [001] direction, owing to the cubic to tetragonal phase transition of $\mathrm{BaTiO}_{3}$. The values for the film are measured along [010] and [001] directions of $\mathrm{BaTiO}_{3}$ substrate, respectively.

the temperature dependent lattice strains of the films in the growth plane follow those of the $\mathrm{BaTiO}_{3}$ substrate at low temperatures, particularly in the temperature range of our experiments $(<400 \mathrm{~K})$, i.e., in-plane lattice clamping by the $\mathrm{BaTiO}_{3}$ substrate. The structural results shown in Table I are consistent with this assumption, specifically the in-plane tetragonal distortion between [010] and [001] directions. Inplane lattice clamping by the substrate is an essential condition for the approach described here, particularly at each of the three structural transitions. Therefore, in-plane strains are controlled by the substrate, and they are the key parameters that strongly influence the out-of-plane component. In order to simplify the discussion of the effects associated with the structural transitions of $\mathrm{BaTiO}_{3}$ substrate, strains in the growth plane are the ones considered here, and they are estimated using the bulk $\mathrm{BaTiO}_{3}$ data (Fig. 1). We also neglect in-plane shears associated with the monoclinic and rhombohedral phases with their respective distortions of $0.2^{\circ}$ and $0.13^{\circ}$. By symmetry, in-plane strains consist of two components, one of which is the even parity $\epsilon_{a}=\frac{1}{2}\left(\epsilon_{x x}+\epsilon_{y y}\right)$, and the other $\epsilon^{*}=\frac{1}{2}\left(\epsilon_{x x}-\epsilon_{y y}\right){ }^{10,11}$ The even parity strain $\epsilon_{a}$ is a measure of in-plane area change, and $\epsilon^{*}$ corresponds to an in-plane distortion away from square symmetry. ${ }^{10,11}$

Strain induced effects on the electrical transport properties at structural transitions of the substrate have been studied using four-terminal measurements. The electrical resistivity of $\mathrm{SrRuO}_{3}$ films grown on (100) $\mathrm{BaTiO}_{3}$ exhibits jumps at temperatures 277 and $183 \mathrm{~K}$ during cooling, and at 281 and $190 \mathrm{~K}$ during warming, as shown in Fig. 2 for a $1000 \AA$ $\mathrm{SrRuO}_{3}$ film. The observed anomalies occur at the temperatures where $\mathrm{BaTiO}_{3}$ substrate also undergoes phase transitions, ${ }^{9}$ indicating that they are caused by the lattice distortions associated with the transitions. The measured resistivity (shown in Fig. 2) increases by $\sim 1 \%$ at the tetragonal to monoclinic transition but decreases by $\sim 5 \%$ at the monoclinic to rhombohedral transition. Both the magnitude and sign of the resistivity changes remain constant through many thermal cycles, indicating that the structural effects are elastic and reversible. In Fig. 2 schematic diagrams of the estimated changes of in-plane symmetry strains at the phase transitions, namely $\epsilon_{a}$ and $\epsilon^{*}$, are also shown. However, the in-plane lattice distortions at the transitions are not simple because of the presence of two possible configurations due to different polings in the (100) plane, so correlations between Downloaded 24 Feb 2007 to 128.104.198.190. Redistribution subject

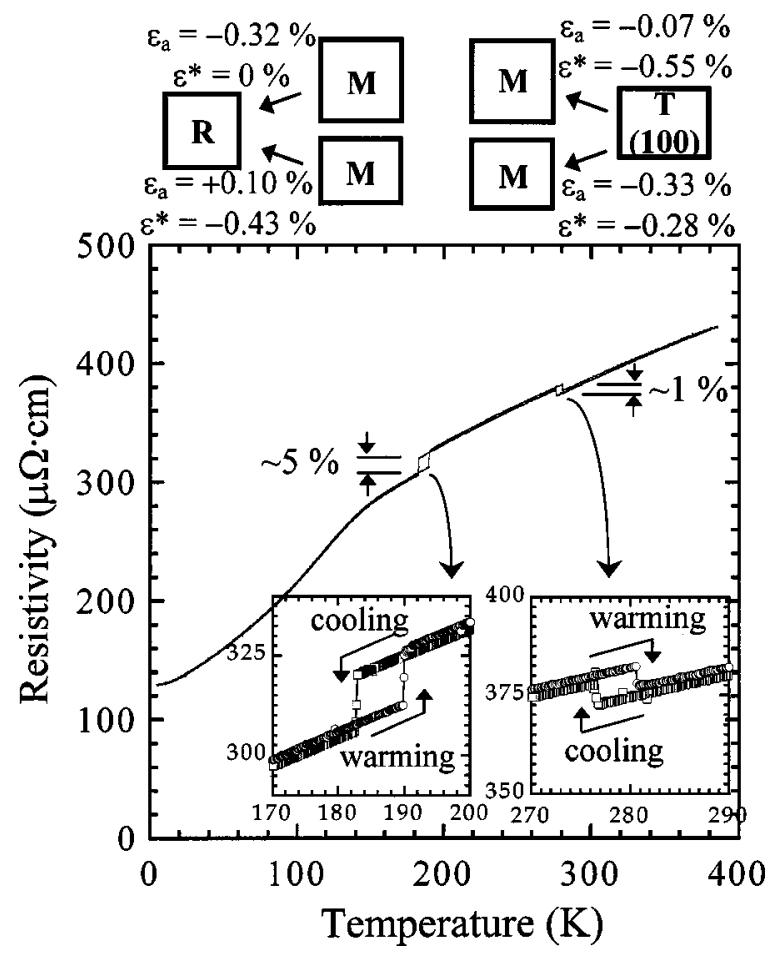

FIG. 2. Electrical resistivity as a function of temperature of a $1000 \AA$ $\mathrm{SrRuO}_{3}$ film grown on (100) $\mathrm{BaTiO}_{3}$ substrate. The lower insets are the zoomed-in view of the thermal hysteresis at the two transitions, and the corresponding in-plane lattice distortions of (100) $\mathrm{BaTiO}_{3}$ are schematically depicted above the graph. The resistivity data were obtained from six thermal cycles.

the resistivity anomalies and lattice strains are difficult. Temperature dependent structural measurements are necessary to elucidate this.

The electrical resistivity of LSMO films grown on (001) $\mathrm{BaTiO}_{3}$ has also been studied, and the results for a $500 \AA$ thick LSMO film are shown in Fig. 3. Similar to the $\mathrm{SrRuO}_{3}$ counterparts, the LSMO films exhibit similar discontinuities in resistivity (Fig. 3). The measured changes are 12\% and $10 \%$ and remain constant over many thermal cycles. Unlike their $\mathrm{SrRuO}_{3}$ counterparts that exhibit a change of sign at the two phase transitions, the resistivity anomalies of the LSMO film do not change the sign.

The lattice structure of the (001) plane of tetragonal $\mathrm{BaTiO}_{3}$ is simpler than that of the (100) plane (Fig. 2), because it is unique through the phase transitions, as is illustrated in the schematic diagrams in Fig. 3. When it undergoes a transition from tetragonal to monoclinic structure, the (001) in-plane lattice undergoes a $0.21 \%$ expansion in $\epsilon_{a}$ accompanied by a $0.26 \%$ distortion in $\epsilon^{*}$. The subsequent transition from monoclinic to rhombohedral induces a $0.10 \%$ expansion in $\epsilon_{a}$ and a $-0.43 \%$ distortion in $\epsilon^{*}$. Recent experiments ${ }^{8}$ show that in-plane biaxial strain can enhance resistivity of $\mathrm{La}_{0.8} \mathrm{Ca}_{0.2} \mathrm{MnO}_{3}$ grown on (001) $\mathrm{SrTiO}_{3}$. The observed resistivity anomalies may have similar origins. One possible mechanism ${ }^{10,11}$ arises from an increase in the JahnTeller splitting of Mn $e_{g}$ level produced by the sudden biaxial tension in the growth plane of the LSMO film, thereby enhancing electron trapping leading to an increase of $\rho$.

Since twins can be easily formed in $\mathrm{BaTiO}_{3}$, one cannot eliminate the possibility of substrate induced cracking in the film completely. Cracking can cause irreversible increase of to AIP license or copyright, see http://apl.aip.org/apl/copyright.jsp 


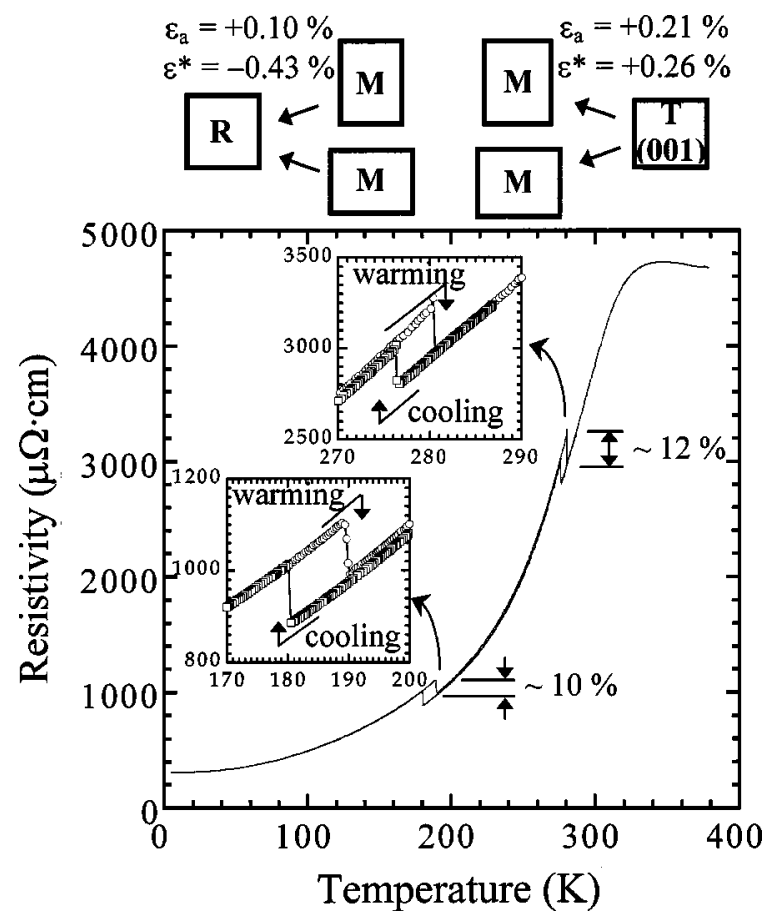

FIG. 3. Electrical resistivity as a function of temperature of a $500 \AA$ $\mathrm{La}_{0.67} \mathrm{Sr}_{0.33} \mathrm{MnO}_{3}$ film grown on (001) $\mathrm{BaTiO}_{3}$ substrate. The insets are the zoomed-in view of the thermal hysteresis at the two transitions, and the corresponding in-plane lattice distortions of $(001) \mathrm{BaTiO}_{3}$ are schematically depicted above the graph. The resistivity data were obtained from six thermal cycles.

resistivity, which in fact has been observed in other samples in this study. However, the reversible behaviors reported here (Figs. 2 and 3) over many thermal cycles indicate that they are intrinsic, not the result of cracks.

Magnetic behavior of the LSMO films grown on $\mathrm{BaTiO}_{3}$ has been studied in the temperature range of $5-400 \mathrm{~K}$ by SQUID magnetometry. The temperature dependent response for a $500 \AA$ LSMO film in a field of 20 Oe applied along the [100] direction is shown in Fig. 4. The observed magnetization exhibits large changes of about $70 \%$ and $10 \%$ at the two transition temperatures. Field-dependent magnetization loops measured above and below the tetragonal to monoclinic transition also show corresponding change in magnetism, As shown in Fig. 4 inset. The magnetization loop measured above the transition is nearly square and has well-defined saturation and coercive fields $\left(H_{s} \sim 250 \mathrm{Oe}\right.$ and $\left.H_{c} \sim 70 \mathrm{Oe}\right)$ consistent with behavior along magnetic easy axis, while the behavior below the transition exhibits much higher saturation field ( $\sim 800$ Oe) and nearly zero remanance indicating hard axis. The observed behavior corresponds to a reversal of magnetic anisotropy owing to lattice distortions induced by the substrate. The observed behavior is consistent with earlier report, ${ }^{11}$ indicating that magnetic anisotropy of LSMO is very sensitive to in-plane lattice distortions.

In summary, biaxial strains induced by the ferroelectric phase transitions of $\mathrm{BaTiO}_{3}$ substrate have been used to modify the electrical transport and magnetic properties of ferromagnetic $\mathrm{SrRuO}_{3}$ and CMR LSMO epitaxial thin films. Large and abrupt changes in resistivity and magnetization of

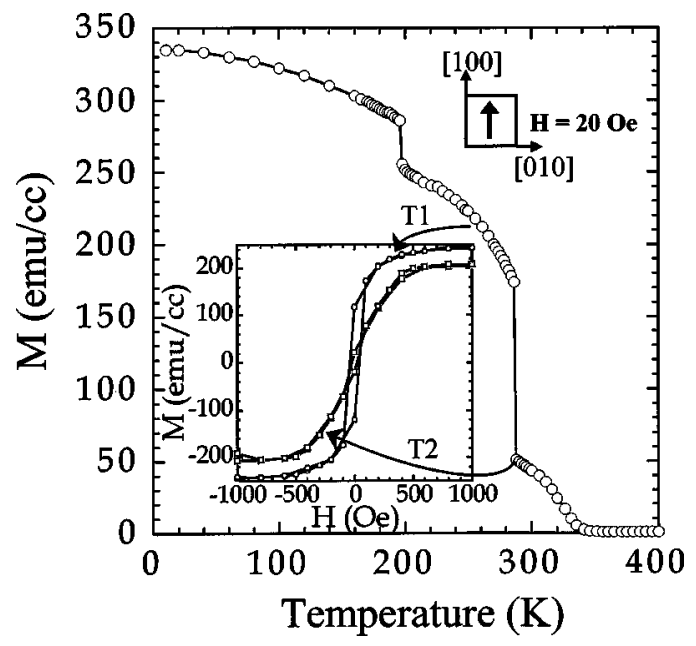

FIG. 4. Magnetization as a function of temperature of a $500 \AA$ $\mathrm{La}_{0.67} \mathrm{Sr}_{0.33} \mathrm{MnO}_{3}$ film grown on (001) $\mathrm{BaTiO}_{3}$ substrate in a magnetic field of 20 Oe applied along [100] direction. The inset shows field dependent hysteresis loops at temperatures above (T1) and below (T2) the phase transition from tetragonal to monoclinic.

both $\mathrm{SrRuO}_{3}$ and LSMO films have been observed at the phase transition temperatures of $\mathrm{BaTiO}_{3}$. This approach provides a controlled way to study strain dependent behavior at the transition temperatures by keeping all other structural parameters fixed, particularly growth conditions, film thickness, and morphology. Direct measurements of threedimensional strain states of the films at these temperatures are also necessary to understand these effects more quantitatively. From a wider perspective, this approach of using ferroelectric substrates promises potential for tuning physical properties of perovskite oxide materials for scientific and device applications. A careful design using this method can produce more reproducible and quantitative results. For instance, biasing fields can be used to alter and control the ferroelectric domain patterns, which can enhance this approach substantially.

This work was supported by Grant Nos. NSF DMR9802444, NSF DMR-9973801, a NSF Young Investigator Award (C. B. E.) and a David and Lucile Packard Fellowship (C. B. E.), and NSF DMR-9703419 (F. T.), and NSF DMR9601825 (F. T.).

${ }^{1}$ H. Sato, M. Naito, and H. Yamamoto, Physica C 280, 178 (1997).

${ }^{2}$ J. P. Locquet, J. Perret, J. Fompeyrine, E. Machler, J. W. Seo, and G. Van Tendeloo, Nature (London) 394, 453 (1998).

${ }^{3}$ W. Si and X. X. Xi, Appl. Phys. Lett. 74, 2839 (1999).

${ }^{4}$ J. O'Donnell, M. S. Rzchowski, J. N. Eckstein, and I. Bozovic, Appl. Phys. Lett. 72, 1775 (1998)

${ }^{5}$ Y. Suzuki, H. Y. Hwang, S.-W. Cheong, and P. R. B. Van Dover, Appl. Phys. Lett. 71, 140 (1997).

${ }^{6}$ Q. Gan, R. A. Rao, C. B. Eom, J. L. Garrett, and M. Lee, Appl. Phys. Lett. 72, 978 (1998).

${ }^{7}$ R. A. Rao, Q. Gan, C. B. Eom, R. J. Cava, Y. Suzuki, J. J. Krajewski, S. C. Gausepohl, and M. Lee, Appl. Phys. Lett. 70, 3035 (1997).

${ }^{8}$ R. A. Rao, D. Lavric, T. K. Nath, C. B. Eom, L. Wu, and F. Tsui, Appl. Phys. Lett. 73, 3294 (1998).

${ }^{9}$ H. F. Kay and P. Vousden, Philos. Mag. 40, 1019 (1949).

${ }^{10}$ A. J. Millis, T. Darling, and A. Migliori, J. Appl. Phys. 83, 1588 (1998).

${ }^{11}$ A. J. Millis,. A. Goyal, M. Rajeswari, K. Gosh, R. Shreekala, R. L. Greene, R. Ramesh, and T. Venkatesan (unpublished); F. Tsui, M. C. Smoak, T. K. Nath, and C. B. Eom, Appl. Phys. Lett. 76, 2421 (2000). 